\title{
Preoperative prognostic nutritional index predicts both short and long-term outcomes after liver resection for hepatocellular carcinoma.
}

Yu Saito ( $\nabla$ saito.yu.1001@tokushima-u.ac.jp )

Tokushima University

Satoru Imura

Department of Surgery, Tokushima University

Yuji Morine

Department of Surgery, Tokushima University

Tetsuya Ikemoto

Department of Surgery, Tokushima University

Shinichiro Yamada

Department of Surgery, Tokushima University

Mitsuo Shimada

Department of Surgery, Tokushima University

Research article

Keywords: prognostic nutritional index, surgical outcomes, hepatocellular carcinoma

Posted Date: July 1st, 2019

DOI: https://doi.org/10.21203/rs.2.10783/v1

License: (c) (1) This work is licensed under a Creative Commons Attribution 4.0 International License.

Read Full License

Version of Record: A version of this preprint was published at Journal of the American College of Surgeons on October 1st, 2017. See the published version at https://doi.org/10.1016/j.jamcollsurg.2017.07.865. 


\section{Abstract}

Background The aim of this study was to investigate the prognostic significance of the prognostic nutritional index (PNI) for both short and long term outcomes after liver resection for hepatocellular carcinoma (HCC). Methods 162 (without any previous treatment) of 229 surgically treated HCC patients were retrospectively analyzed. The cut off value of the preoperative PNI was 45.0. Patients were divided into two groups, $\mathrm{PNI}$ low $(n=76)$ and high $(n=86)$ group. Results Among some immune parameters such as $\mathrm{PNI}$, neutrophil to lymphocyte ratio (NLR) and aspartate aminotransferase (AST) to lymphocyte ratio (ALRI), PNI had most reliable parameters in terms with prediction of both short and long term outcomes. Preoperative PNI tended to correlate with low skeletal muscle mass (SMM). In short term outcomes, PNI low group were more likely to have postoperative complications. The disease-free survival rate in PNI low group was significantly worse than that in the PNI high group ( 20.5 vs. $48.7 \%, 5$ year SR, $p=0.03$ ). On multivariate analysis, Low PNI was an independent prognostic factor for disease free survival (HR 1.65, $p=0.04$ ). Conclusions The preoperative PNI was the most significant prognostic factor for evaluating both short and long-term outcomes after liver resection for HCC.

\section{Background}

In liver resection $(\mathrm{Hx})$ for hepatocellular carcinoma $(\mathrm{HCC})$, the perioperative complications and mortality has been improved recent years, however, it has been critical to manage patients with various comorbidity. Furthermore, the recurrent rate after curative $\mathrm{Hx}$ for $\mathrm{HCC}$ is still higher than other digestive organ cancers and it has been also important to acsses risk factors for recurrence after curative $\mathrm{Hx}$.

PNI (Prognostic nutritional index) was firstly reported in 1980 by Buzby et al. ${ }^{1)}$ in order to predict perioperative risk. However, calculation method was too complicated using many parameters such as serum albumin (Alb), triceps skinfold (TSF), transferrin (TFN) and delayed hypersensitivity skin testing (DHS). 4 years later, Japanese Onodera et al. ${ }^{2)}$ reported simpler modified PNI using serum Alb and total lymphocytes count (TLC) alone, and that Onodera's PNI has been widely used for perioperative risk assessment. In addition to perioperative risk prediction, PNI was also reported that it correlated with longterm prognosis of various cancers after curative treatment such as lung ${ }^{3)}$, ovarian ${ }^{4}$ ), cervical ${ }^{5}$ ), gastric ${ }^{6}$ ) and colorectal ${ }^{7)}$ cancers and so on.

In Hx for HCC, preoperative PNI was already reported to correlate with liver function such as albuminbilirubin (ALBI) grade, and to predict short-term outcomes after $\mathrm{Hx}$ for $\mathrm{HCC}$ within the Milan criteria ${ }^{8}$. Chan et al. ${ }^{9)}$ reported that preoperative PNI predicted long-term prognosis after $\mathrm{Hx}$ in only early BCLC stage HCC. There were no reports of preoperative PNI for predicting both short and long term outcomes in whole HCC stage.

In the present study, among various immune parameters such as PNI, neutrophil to lymphocyte ratio (NLR) and aspartate aminotransferase (AST) to lymphocyte ratio (ALRI), the most reliable parameter was 
examined. The aim of this study was to investigate the prognostic significance of the preoperative PNI for both short and long term outcomes after $\mathrm{Hx}$ for whole stage HCC patients.

\section{Methods \\ Patients}

Among 229 patients who underwent Hx from January 2006 to December 2014, 162 patients were enrolled in this study. Inclusion criteria of this study were 1) Primary $\mathrm{Hx}, 2$ ) No any other treatments before $\mathrm{Hx}, 3$ ) Availability of follow up data. The study was approved by Tokushima University Hospital ethics committee and with the approval of corresponding regulatory agencies, and all the experiments were carried out in accordance with the approved guidelines (Tokushima Clinical Trial Management System Number; 3215). Meanwhile, all the patients involved in the study signed the informed consent form and agreed to participate.

\section{Preoperative immune parameters}

Blood samples were taken prior to $\mathrm{Hx}$. The PNI was the sum of serum albumin and $0.005 \times$ lymphocyte count. The NLR was calculated by dividing neutrophil count by lymphocyte count. ALRI was calculated by dividing AST by lymphocyte count. The cutoff value of PNI, NLR and ALRI was 45, 2.3 and 30.8 calculated by receiver-operating characteristic (ROC) curve for predicting recurrence after $\mathrm{Hx}$.

\section{Assessment of fat mass (FM) and skeletal muscle mass (SMM)}

Preoperative fat mass (FN) or skeletal muscle mass (SMM) was investigated from CT modality using Synapse Vincent ${ }^{\circledR}$. Visceral FM $\left(\mathrm{cm}^{2}\right)$, subcutaneous FM $\left(\mathrm{cm}^{2}\right)$ and

$\mathrm{SMM} /$ height $\left(\mathrm{cm}^{2} / \mathrm{m}^{2}\right)$ were automatically calculated.

\section{Follow-up after $\mathrm{Hx}$}

Monthly follow-up was conducted by assessment of tumor markers (AFP, DCP, and AFP-L3) and ultrasonography. Dynamic computed tomography (CT) scan and Gd-EOB-DTPA-enhanced magnetic resonance imaging (MRI) were conducted at 3 and 6 months post operation. We defined recurrence as the appearance of new lesions with radiological features typical of HCC, as confirmed by at least two imaging methods.

\section{Statistical analysis}


All statistical analysis was performed using SPSS Version 21.0 statistical software (SPSS, Chicago, IL). A $p$-value of less than 0.05 was considered statistically significant. Relationships between PNI and the clinicopathological variables were analyzed using the chi-square test and Mann-Whitney U test. Survival curves were calculated using the Kaplan-Meier method and compared with the log-rank test. All factors significant by univariate analysis were included in the Cox's proportional hazards model of multivariate analysis to identify independent factors influencing survival. The factors included for analyses were patient age (under 70 / over 70 y.o.), gender (male / female), HBsAg (absent / present), HCVAb (absent / present), AFP (under 200 / over $200 \mathrm{ng} / \mathrm{ml}$ ), DCP (under 400/over $400 \mathrm{mAU} / \mathrm{ml} /$ ), yumor number (single / multiple), tumor size (under 3 / over $3 \mathrm{~cm}$ ), tumor differentiation (well, moderate / poor), portal invasion (absent / present), staging (I, / /, $\mathbb{Q}$, ) and PNI (high / low).

\section{Results}

\section{Comparison of immune parameters among PNI, NLR and ALRI}

Table 1 showed the comparison in both short and long-term outcomes among PNI, NLR and ALRI. The PNI alone predicted short-term outcomes, and both the PNI and NLR were reliable parameters for predicting the long-term outcomes. In terms with prediction of both short and long-term outcomes, PNI was most reliable parameters among three parameters.

\section{Correlation between preoperative PNI and the clinicopathological variables}

Correlations between preoperative PNI values and clinicopathological variables are shown in Table 2 . Low PNI significantly correlated with high age, or impaired liver function such as higher AST, higher ICGR15 and lower platelet levels. In tumor factors, low PNI tended to correlate with the only presence of microscopic vp alone. Furthermore, low PNI significantly correlated with lower SMM, which was called Sarcopenia.

\section{Preoperative PNI and short-term outcomes}

There were no significant differences in operative procedures between PNI low and high groups. Blood loss was significant much more in Low PNI group. Low PNI group tended to have more frequent severe postoperative complications, and had significant longer hospital stays after $\mathrm{Hx}$ (Table 3 ).

\section{Preoperative PNI and long-term outcomes}


In overall survival (OS), Low PNI group tended to have worse prognosis (Figure 1A). In disease free survival (DFS), Low PNI group had significant worse prognosis than high PNI group (Figure 1B). In univariate analysis for DFS, high AFP, high DCP, multiple tumors, the presence of microvascular invasion, more advanced stage and low PNI were selected for poor prognostic factors. In multivariate analysis revealed that high AFP, multiple tumors and low PNI were independent prognostic factors for DFS (Table 4). Regarding recurrent patterns, there was no significant difference between PNI low and high group (Figure 2).

\section{Discussion}

In the present study, some immune parameters including PNI, NLR and ALR were compared in terms with both short and long term outcome, and PNI was the most reliable parameters. In addition to the prediction of outcomes after HX, PNI significantly correlated with low SMM, Sarcopenia. This is the first reports of preoperative PNI for predicting both short and long term outcomes in whole HCC stage.

First of all, there were several reports about NLR for prognostic factors in HCC after curative treatment. He et al. ${ }^{10)}$ reported the usefulness of NLR and platelets to lymphocytes ratio (PLR) after transarterial chemoembolization. Taussig et al. also reported ${ }^{11)}$ thatNLR predicted disease progression following intra-arterial therapy of HCC. In the present study, NLR was good biomarkers for predicting long-term outcomes after $\mathrm{Hx}$. The molecular mechanism of an elevated NLR involved many factors and it remains poorly understood. However, a close relationship between the accumulation of tumor-associated macrophages in HCC and high NLR values has been observed in patients with HCC who underwent hepatic resection and living-donor liver transplantation ${ }^{12}$. A high NLR was also associated with a high infiltration of tumor-associated macrophages and high inflammatory cytokine production in the tumor, such as interleukin-6, interleukin-8 and interleukin-17, which promote systemic neutrophilia ${ }^{13), 14)}$.

On the other hand, NLR was not correlated with short-term outcomes in the present study. PNI was only predictors for postoperative complications and hospital stays. PNI value, a combination of the albumin and total lymphocyte count, was parameters to evaluate the immunological and nutritional aspects of patients undergoing surgery. Ke et al. ${ }^{15)}$ reported that PNI was constructed as a reflection of a patient's nutritional status and it made sense that the PNI might be related to postoperative complications. The PNI included the lymphocyte count in its calculation. It has been found that the level of serum Alb and the count of lymphocyte had a tight relationship with the induction of the inflammatory response ${ }^{16)}$. Therefore, it not only reflected the status of nutrition but also systemic inflammation.

For patients with a low PNI, it was essential to improve their outcomes through perioperative nutritional interventions, for example, the administration of branched-chain amino acid-enriched nutrient support ${ }^{17)}$, 18). In the present study, PNI significantly correlated with Sarcopenia, so further nutritional intervention might be necessary for patients with a low PNI. 


\section{Conclusions:}

Low PNI correlated with Sarcopenia reflecting low nutritional and inflammatory response. Furthermore, preoperative PNI, rather than NLR and ALRI was the most significant and reliable prognostic factor for evaluating both short and long-term outcomes after Hx for HCC. Some nutritional interventions might be necessary for the patients with a low PNI.

\section{Abbreviations}

Prognostic nutritional index; PNI

Liver resection; $\mathrm{Hx}$

Hepatocellular carcinoma; HCC

Neutrophil to lymphocyte ratio; NLR

Aspartate aminotransferase to lymphocyte ratio; ALRI

indocyanine green retention test; ICG R15

Area under curve; AUC

Receiver operating characteristic; ROC

\section{Declarations}

\section{Ethics approval and consent to participate}

The study was approved by Tokushima University Hospital ethics committee and with the approval of corresponding regulatory agencies, and all the experiments were carried out in accordance with the approved guidelines (Tokushima Clinical Trial Management System Number; 3215). Meanwhile, all the patients involved in the study signed the informed consent form and agreed to participate.

Consent for publication

Not applicable

\section{Availability of data and materials}

The datasets generated and/or analyzed during the current study are not publicly available due [No informed consent of publicly availability] but are available from the corresponding author on reasonable request. 


\section{Competing interests}

All authors declare that they have no competing interests.

\section{Funding}

All authors declare that they have no sources of funding for the research.

\section{Authors' contributions}

Yu Saito (YS) MD, PhD, FACS: Participated in the research design, performance of the research, data analysis and writing manuscripts.

Satoru Imura (SI) MD, PhD, FACS: Participated in the research design and data analysis.

Yuji Morine (YM) MD, PhD, FACS: Participated in the research design.

Tetsuya Ikemoto (TI) MD, PhD, FACS: Participated in the research design.

Shinichiro Yamada (SY) MD, PhD, FACS: Participated in data analysis.

Mitsuo Shimada (MS) MD, PhD, FACS: Participated in the critical comments and administrative support.

\section{Acknowledgements}

Not applicable

\section{References}

1. Buzby GP, Mullen JL, Matthews DC, Hobbs CL, Rosato EF. Prognostic nutritional index in gastrointestinal surgery. Am J Surg. 1980 Jan;139(1):160-7.

2. Onodera T, Goseki N, Kosaki G. Prognostic nutritional index in gastrointestinal surgery of malnourished cancer patients. Nihon Geka Gakkai Zasshi. 1984 Sep;85(9):1001-5.

3. Okada S, Shimada J, Kato D, Tsunezuka H, Teramukai S, Inoue M. Clinical Significance of Prognostic Nutritional Index After Surgical Treatment in Lung Cancer. Ann Thorac Surg. 2017 Jul;104(1):296302.

4. Miao Y, Li S, Yan Q, Li B, Feng Y. Prognostic Significance of Preoperative Prognostic Nutritional Index in Epithelial Ovarian Cancer Patients Treated with Platinum-Based Chemotherapy. Oncol Res Treat. 2016;39(11):712-719. 
5. Haraga J, Nakamura K, Omichi C, Nishida T, Haruma T, Kusumoto T, et al. Pretreatment prognostic nutritional index is a significant predictor of prognosis in patients with cervical cancer treated with concurrent chemoradiotherapy. Mol Clin Oncol. 2016 Nov;5(5):567-574.

6. Han WX, Chen ZM, Wei ZJ, Xu AM. Preoperative pre-albumin predicts prognosis of patients after gastrectomy for adenocarcinoma of esophagogastric junction. World J Surg Oncol. 2016 Nov 3;14(1):279.

7. Yang Y, Gao P, Chen X, Song Y, Shi J, Zhao J, et al. Prognostic significance of preoperative prognostic nutritional index in colorectal cancer: results from a retrospective cohort study and a meta-analysis. Oncotarget. 2016 Sep 6;7(36):58543-58552.

8. Ke M, Xu T, Li N, Ren Y, Shi A, Lv Y, et al. Prognostic nutritional index predicts short-term outcomes after liver resection for hepatocellular carcinoma within the Milan criteria. Oncotarget. 2016 Dec 6;7(49):81611-81620.

9. Chan AW, Chan SL, Wong GL, Wong VW, Chong CC, Lai PB, et al. Prognostic Nutritional Index (PNI) Predicts Tumor Recurrence of Very Early/Early Stage Hepatocellular Carcinoma After Surgical Resection. Ann Surg Oncol. 2015 Dec;22(13):4138-48.

10. He CB, Lin XJ. Inflammation scores predict the survival of patients with hepatocellular carcinoma who were treated with transarterial chemoembolization and recombinant human type- 5 adenovirus H101. PLoS One. 2017 Mar 29;12(3):e0174769

11. Taussig MD, Irene Koran ME, Mouli SK, Ahmad A, Geevarghese S, Baker JC, et al. Neutrophil to lymphocyte ratio predicts disease progression following intra-arterial therapy of hepatocellular carcinoma. HPB (Oxford). 2017 May;19(5):458-464.

12. Shirabe K, Mano Y, Muto J, Matono R, Motomura T, Toshima T, et al. Role of tumor-associated macrophages in the progression of hepatocellular carcinoma. Surg Today. 2012;42:1-7.

13. Harimoto N, Shirabe K, Nakagawara H, Toshima T, Yamashita Y, Ikegami T, et al. Prognostic factors affecting survival at recurrence of hepatocellular carcinoma after living-donor liver transplantation: with special reference to neutrophil/lymphocyte ratio. Transplantation. 2013;96:1008-12.

14. Peng W, Li C, Wen TF, Yan LN, Li B, Wang WT, et al. Neutrophil to lymphocyte ratio changes predict small hepatocellular carcinoma survival. J Surg Res. 2014;192:402-8.

15. Ke M, Xu T, Li N, Ren Y, Shi A, Lv Y, et al. Prognostic nutritional index predicts short-term outcomes after liver resection for hepatocellular carcinoma within the Milan criteria. Oncotarget. 2016 Dec 6;7(49):81611-81620.

16. Koike Y, Miki C, Okugawa Y, Yokoe T, Toiyama Y, Tanaka K, et al. Preoperative C-reactive protein as a prognostic and therapeutic marker for colorectal cancer. J Surg Oncol. 2008;98:540-544.

17. Okabayashi T, Nishimori I, Sugimoto T, Maeda H, Dabanaka K, Onishi S, et al. Effects of branchedchain amino acids-enriched nutrient support for patients undergoing liver resection for hepatocellular carcinoma. J Gastroenterol Hepatol. 2008;23:1869-1873.

18. Okabayashi T, Nishimori I, Nishioka A, Yamashita K, Sugimoto T, Dabanaka K, et al. Long-term effects of multimodal treatment for patients with resectable carcinoma of the pancreas. Oncol Rep. 


\section{Tables}

Table 1₫Comparison of immune parameters in short and long-term outcomes

\begin{tabular}{lcccccc}
\hline & Cut off & \multicolumn{2}{c}{ Incidence of complications } & $p$ value & $\begin{array}{c}\text { Hospital } \\
\text { Stays (days) }\end{array}$ & $p$ value \\
& & & & & & \\
\hline PNI & 45.0 & Low & $8.2 \%$ & 0.12 & $31 \pm 34$ & $\leq 0.01$ \\
& & High & $2.7 \%$ & & $17 \pm 9$ & \\
\hline NLR & 2.3 & Low & $5.7 \%$ & 0.98 & $21 \pm 16$ & 0.13 \\
& & High & $5.6 \%$ & & $27 \pm 33$ & \\
\hline ALRI & 30.8 & Low & $6.5 \%$ & 0.87 & $23 \pm 34$ & 0.67 \\
& & High & $5.8 \%$ & & $25 \pm 18$ & \\
\hline
\end{tabular}

\begin{tabular}{lcccccc}
\hline & Cut off & & OS 3y SR & $p$ value & DFS 3y SR & $p$ value \\
\hline PNI & 45.0 & Low & $68.4 \%$ & 0.06 & $38.2 \%$ & $\underline{0.03}$ \\
& & High & $79.1 \%$ & & $53.7 \%$ & \\
\hline NLR & 2.3 & Low & $83.9 \%$ & $\underline{0.01}$ & $49.5 \%$ & $\underline{0.04}$ \\
& & High & $65.7 \%$ & & $40.5 \%$ & \\
\hline ALRI & 30.8 & Low & $77.8 \%$ & 0.25 & $51.6 \%$ & 0.25 \\
& & High & $71.5 \%$ & & $41.5 \%$ & \\
\hline
\end{tabular}

Table 2: Correlation between preoperative PNI and the clinicopathological variables 


\begin{tabular}{|c|c|c|c|}
\hline \multirow{3}{*}{$\begin{array}{l}\text { Factors } \\
\text { Age: Mean } \\
\text { Genger: }\end{array}$} & \multicolumn{2}{|c|}{ Preoperative PNI } & \multirow[b]{2}{*}{$p$-value } \\
\hline & $<45(n=86)$ & $\geqq 45(n=76)$ & \\
\hline & $70 \pm 10$ & $60 \pm 10$ & $\leq 0.01$ \\
\hline $\begin{array}{l}\text { Male / Female } \\
\text { HBs Ag: }\end{array}$ & $57 / 29$ & $62 / 14$ & $\underline{0.03}$ \\
\hline $\begin{array}{l}\text { absent / present } \\
\text { HCV Ab: }\end{array}$ & $67 / 19$ & $57 / 19$ & 0.66 \\
\hline $\begin{array}{l}\text { absent / present } \\
\text { AST (IU / I): Mean } \\
\text { PT (\%): Mean } \\
\text { T-bil (mg / dl): Mean } \\
\text { ICG R15 (\%): Mean } \\
\text { Platelet: }\end{array}$ & $\begin{array}{c}48 / 38 \\
60 \pm 36 \\
100 \pm 17 \\
0.9 \pm 0.4 \\
16 \pm 10\end{array}$ & $\begin{array}{c}48 / 28 \\
42 \pm 24 \\
103 \pm 22 \\
0.8 \pm 0.3 \\
11 \pm 8\end{array}$ & $\begin{array}{c}0.34 \\
<0.01 \\
0.31 \\
0.19 \\
\leq 0.01\end{array}$ \\
\hline $\begin{array}{l}</ \geqq 10 \times 10^{4} \\
\text { Tumor size: Mean } \\
\text { Tumor number: }\end{array}$ & $\begin{array}{c}21 / 65 \\
4.9 \pm 4.5\end{array}$ & $\begin{array}{c}4 / 72 \\
4.1 \pm 2.7\end{array}$ & $\frac{<0.01}{0.18}$ \\
\hline $\begin{array}{l}\text { single / multiple } \\
\text { vp: }\end{array}$ & $60 / 26$ & $57 / 19$ & 0.46 \\
\hline $\begin{array}{l}\text { absent / present } \\
\text { im: }\end{array}$ & $61 / 24$ & 65 / 11 & 0.06 \\
\hline $\begin{array}{l}\text { absent / present } \\
\text { Tumor differentiation: }\end{array}$ & $79 / 7$ & $70 / 6$ & 0.65 \\
\hline $\begin{array}{l}\text { well, moderate / poor } \\
\text { Staging: }\end{array}$ & 17 / 69 & 19 / 57 & 0.47 \\
\hline $\begin{array}{l}\text { I, II / III, IV } \\
\text { AFP: }\end{array}$ & $57 / 29$ & $53 / 23$ & 0.73 \\
\hline $\begin{array}{l}</ \geqq 200 \mathrm{ng} / \mathrm{ml} \\
\mathrm{DCP}:\end{array}$ & 69 / 17 & 59 / 17 & 0.69 \\
\hline $\begin{array}{l}</ \geqq 400 \mathrm{mAU} / \mathrm{ml} \\
\text { BMI: Mean } \\
\text { Visceral FM }\left(\mathrm{cm}^{2}\right): \text { Mean }\end{array}$ & $\begin{array}{l}52 / 34 \\
22 \pm 3 \\
76 \pm 47\end{array}$ & $\begin{array}{l}49 / 27 \\
23 \pm 3 \\
87 \pm 47\end{array}$ & $\begin{array}{l}0.60 \\
0.43 \\
0.11\end{array}$ \\
\hline Subcutaneous FM $\left(\mathrm{cm}^{2}\right)$ : Mean & $108 \pm 77$ & $108 \pm 64$ & 0.97 \\
\hline SMM / height $\left(\mathrm{cm}^{2} / \mathrm{m}^{2}\right):$ Mean & $51 \pm 13$ & $56 \pm 15$ & $\underline{0.03}$ \\
\hline
\end{tabular}

Table 3: Preoperative PNI and short-term outcomes 


\begin{tabular}{|c|c|c|c|}
\hline \multirow{2}{*}{ Factors } & \multicolumn{2}{|c|}{ Preoperative PNI } & \multirow{2}{*}{$p$-value } \\
\hline & $<45(\mathrm{n}=86)$ & $\geqq 45(n=76)$ & \\
\hline $\begin{array}{l}\text { Operative factors } \\
\text { Operative procedures: }\end{array}$ & & & \\
\hline $\begin{array}{l}\text { Hr0 / S / } 1 \text { / } 2 \text { / } 3 \\
\text { Operative time (min): Mean } \\
\text { Blood loss (ml): Mean } \\
\text { Transfusion }\end{array}$ & $\begin{array}{c}29 / 4 / 22 / 10 / 1 \\
328 \pm 93 \\
394 \pm 433\end{array}$ & $\begin{array}{c}17 / 25 / 18 / 15 / 1 \\
318 \pm 85 \\
253 \pm 224\end{array}$ & $\begin{array}{l}0.65 \\
0.48 \\
\leq 0.01 \\
\end{array}$ \\
\hline absent / present & $75 / 11$ & $63 / 12$ & 0.48 \\
\hline $\begin{array}{l}\text { Post-operative factors } \\
\text { Complications }\end{array}$ & & & \\
\hline $\begin{array}{l}\text { absent / present } \\
\text { Liver failure } \\
\text { Intra-abdominal abscess } \\
\text { Resistant ascites } \\
\text { Hospital stays (days): Mean }\end{array}$ & $\begin{array}{r}79 / 7 \\
3 \\
3 \\
1 \\
31 \pm 34 \\
\end{array}$ & $\begin{array}{c}74 / 2 \\
0 \\
1 \\
1 \\
17 \pm 9 \\
\end{array}$ & $\leq 0.01$ \\
\hline
\end{tabular}

Table 4: Univariate and Multivariate analysis of DFS

\begin{tabular}{lccccc}
\hline & Univariate & & \multicolumn{3}{c}{ Multivariate } \\
\cline { 2 - 4 } \cline { 5 - 6 } & $p$-value & & Hazard ratio & $95 \%$ C.I. & $p$-value \\
\hline$\geqq 200 \mathrm{ng} / \mathrm{ml}$ & $\leq 0.01$ & & 1.77 & $1.00-3.13$ & 0.04 \\
$\geqq 400 \mathrm{mAU} / \mathrm{ml}$ & 0.04 & & 1.04 & $0.59-1.84$ & 0.89 \\
ər number: multiple & $\leq 0.01$ & & 2.09 & $1.25-3.51$ & $<0.01$ \\
resent & $<0.01$ & & 1.39 & $0.79-2.44$ & 0.25 \\
$\leq 45$ & 0.03 & & 1.65 & $1.00-2.71$ & $\underline{0.04}$ \\
\hline
\end{tabular}

\section{Figures}




\section{Overall survival (OS)}

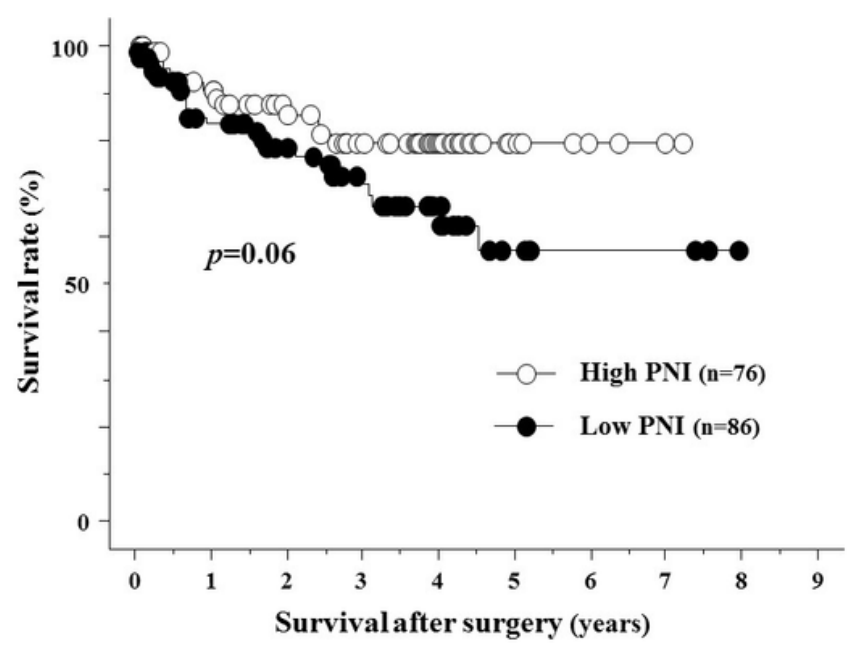

Fig. 1A
Disease free survival (DFS)

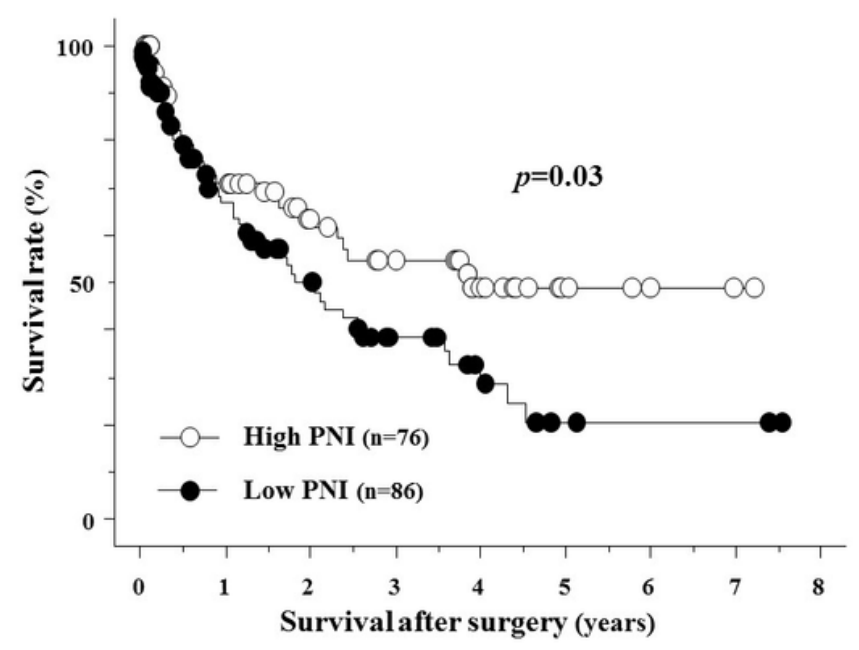

Fig. 1B

\section{Figure 1}

Long-term survival A. Overall survival Low PNI group tended to have worse prognosis $(p=0.06)$. B.

Disease free survival Low PNI group had significant worse prognosis than high PNI group (20.5 vs. 48.7 $\%, 5$ year $\mathrm{SR}, \mathrm{p}=0.03)$. 

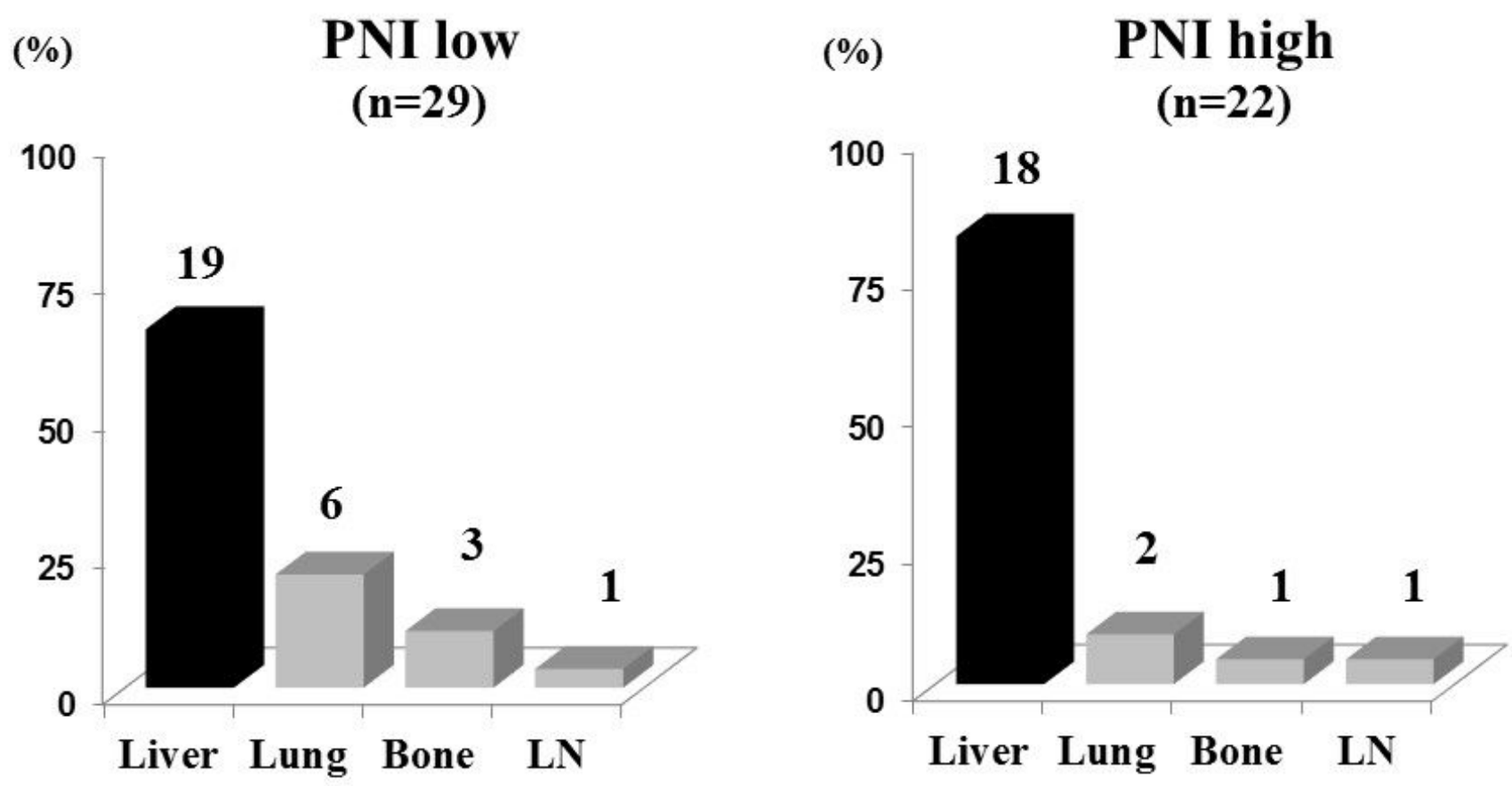

$p=0.67$

LN : lymph node

Fig. 2

Figure 2

Recurrence patterns There was no significant difference in recurrent patterns. 